\title{
Wt1 ablation and Igf2 upregulation in mice result in Wilms tumors with elevated ERK1/2 phosphorylation
}

\author{
Qianghua Hu, ${ }^{1}$ Fei Gao, ${ }^{1}$ Weihua Tian, ${ }^{1}$ E. Cristy Ruteshouser, ${ }^{1}$ Yaqing Wang, ${ }^{2}$ Alexander Lazar, ${ }^{3}$ \\ John Stewart, ${ }^{3}$ Louise C. Strong, ${ }^{1}$ Richard R. Behringer, ${ }^{1,4}$ and Vicki Huff 1,4,5

\begin{abstract}
1Department of Genetics, ${ }^{2}$ Department of Experimental Therapeutics, and ${ }^{3}$ Department of Pathology, University of Texas M.D. Anderson Cancer Center, Houston, Texas, USA. ${ }^{4}$ Graduate Program in Genes and Development and ${ }^{5}$ Graduate Program in Human Molecular Genetics,
\end{abstract} \\ UT-Houston Graduate School of Biomedical Sciences, Houston, Texas, USA.
}

\begin{abstract}
Wilms tumor (WT) is a genetically heterogeneous childhood kidney tumor. Several genetic alterations have been identified in WT patients, including inactivating mutations in WT1 and loss of heterozygosity or loss of imprinting at 11p15, which results in biallelic expression of IGF2. However, the mechanisms by which one or a combination of genetic alterations results in tumorigenesis has remained challenging to determine, given the lack of a mouse model of WT. Here, we engineered mice to sustain mosaic, somatic ablation of Wt 1 and constitutional $\operatorname{Igf} 2$ upregulation, mimicking a subset of human tumors. Mice with this combination of genetic alterations developed tumors at an early age. Mechanistically, $W t 1$ ablation blocked mesenchyme differentiation, and increased $I g f 2$ expression upregulated ERK1/2 phosphorylation. Importantly, a subset of human tumors similarly displayed upregulation of ERK1/2 phosphorylation, which suggests ERK signaling might contribute to WT development. Thus, we have generated a biologically relevant mouse model of WT and defined one combination of driver alterations for WT. This mouse model will provide a powerful tool to study the biology of WT initiation and progression and to investigate therapeutic strategies for cancers with IGF pathway dysregulation.
\end{abstract}

\section{Introduction}

Wilms tumor (WT) is a childhood kidney tumor that is thought to arise from undifferentiated metanephric mesenchyme. WT is genetically heterogeneous. Mutations that occur in tumors include inactivation of WT1 ( $20 \%$ of tumors), somatic stabilizing CTNNB1 mutations ( 15\%), somatic deletion of WTX $(\sim 20 \%)$, and p53 mutations $(\sim 5 \%)$ that occur specifically in the subset of anaplastic WT (1-5). Overall, only one-third of tumors have mutations in 1 or more of these 4 genes (6). Additionally, loss of heterozygosity (LOH) or loss of imprinting (LOI) at the chromosomal region $11 \mathrm{p} 15$, which harbors a cluster of imprinted genes, is observed in approximately $70 \%$ of tumors $(7,8)$, resulting in biallelic expression of IGF2. However, the mechanism by which one or a combination of alterations results in tumorigenesis is not known. Children heterozygous for germline WT1 mutations are predisposed to WT, and these tumors have invariably sustained mutation of the wild-type WT1 allele. However, inactivation of WT1 is also observed in premalignant lesions (9), which suggests that one or more additional, rate-limiting genetic alterations is required for progression to a malignant phenotype.

The cellular pathways dysregulated in WTs as a result of WT1 ablation or IGF2 upregulation are unknown, and identifying such pathways in human tumors is challenging because of the genetic heterogeneity of the disease and the biologic complexity of primary human tumors. While animal models can be powerful tools for dissecting the biology of human tumors, the development of a mouse model for WT has been elusive. $\mathrm{Wt} 1^{-/-}$mice lack kidneys

Authorship note: Qianghua Hu and Fei Gao contributed equally to this work. Conflict of interest: The authors have declared that no conflict of interest exists. Citation for this article: J Clin Invest. 2011;121(1):174-183. doi:10.1172/JCI43772. and die pre- or perinatally $(10,11)$. Unlike $W T 1^{+/-}$children, $W t 1^{+/}$ mice do not develop tumors. While this disparity could be due to intrinsic differences in kidney biology, we hypothesized that 2 major differences between mice and humans account for the lack of WT development in $\mathrm{Wt}^{+/ /}$mice. First, the number of cells at risk for developing WT is much fewer in mice, and thus the loss of the wild-type allele in the undifferentiated metanephric mesenchyme of $W t 1^{+--}$mice is stochastically very rare. Second, in humans, WT1 and the imprinted IGF2 gene are both on the short arm of chromosome 11. LOH for $11 \mathrm{p}$ markers is observed in a subset of WTs and is often due to genetic mechanisms (somatic recombination, chromosome loss, and reduplication) that do not result in a net loss of genetic material (12). Furthermore, in these cases of $11 p$ $\mathrm{LOH}$, retention of paternally derived alleles is invariably observed (13). Therefore, the outcome of $11 \mathrm{p} \mathrm{LOH}$ is often paternal isodisomy. Because of the synteny of IGF2 and WT1, the reduction to homozygosity of a paternally mutant WT1 allele will often result in 2 copies of the paternally expressed IGF2 allele. Indeed, in a study of 36 WTs carrying WT1 mutations, 16 (44\%) displayed IGF2 paternal isodisomy (14). In mice, $W t 1$ and $I g f 2$ are not syntenic. Thus, genetic changes that are accomplished by a single event in humans will, in mice, require 2 independent events, further reducing the probability that the necessary genetic alterations for tumorigenesis would occur in the developing mouse kidney.

To generate an endogenous mouse model for WT, we therefore sought to somatically inactivate WT1 in a small proportion of the differentiating metanephric mesenchyme and in the context of IGF2 biallelic expression. This strategy would allow for the development of functional kidneys (and thus postnatally viable mice) and would also mimic alterations observed in human tumors. For Wt1 ablation, we used the $W t 1^{-}$strain (10) and our conditional 
A

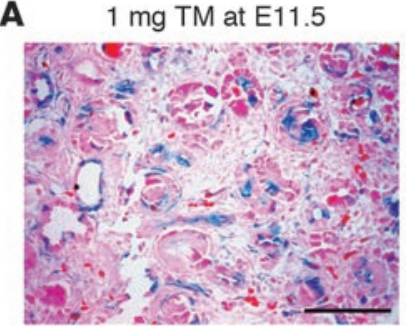

C
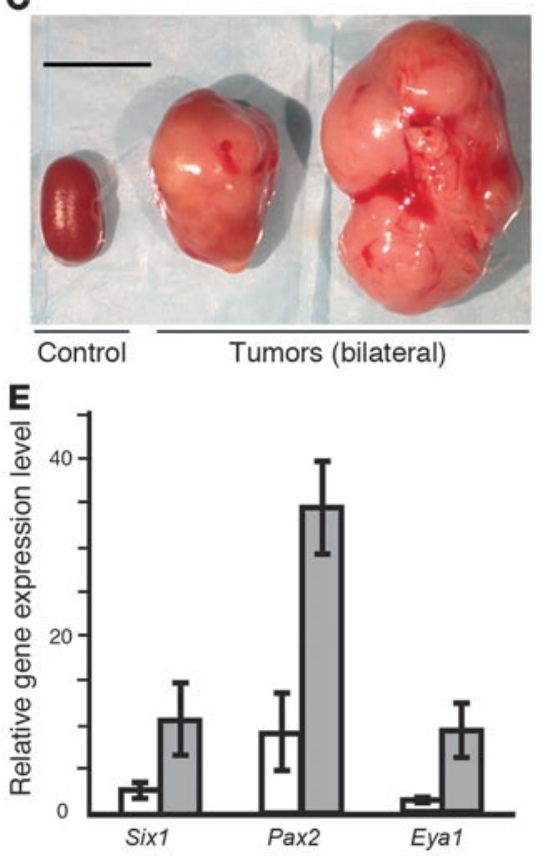
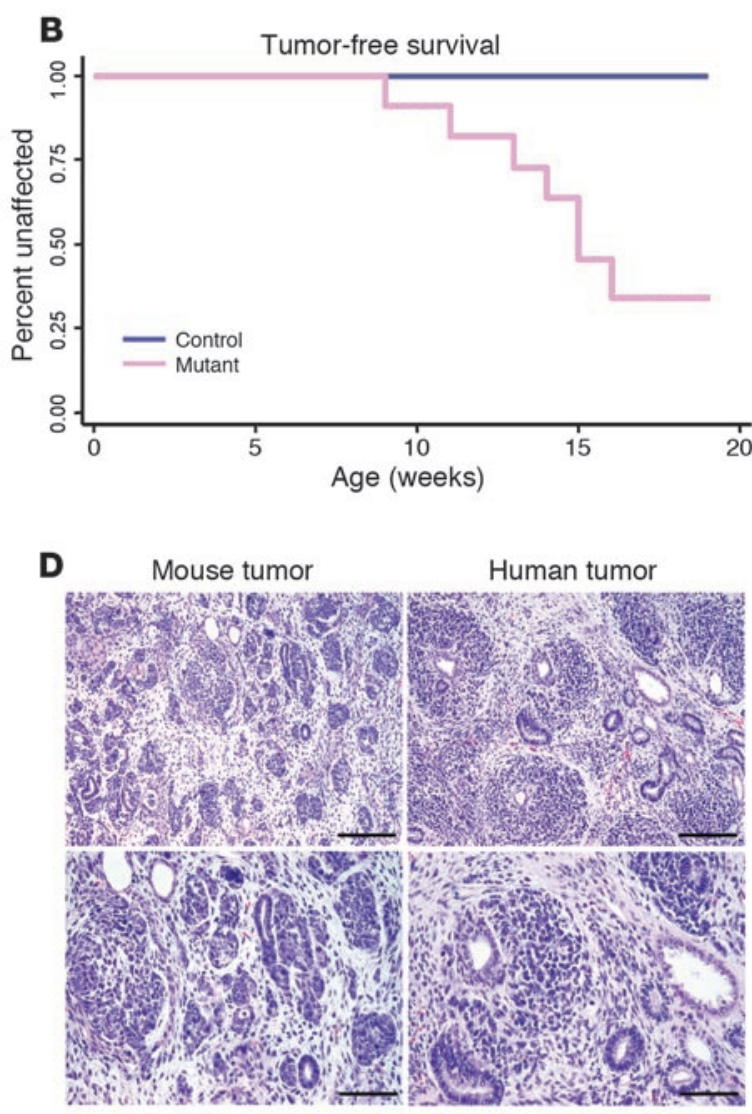

Figure 1

WT development in Wt1-lgf2 mice. (A) Estimation by X-gal staining of Cre-mediated recombination in the kidney after TM induction at E11.5. (B) KaplanMeier plot of tumor-free survival of Wt1-lgf2 and littermate control animals. (C) Bilateral tumors from Wt1-Igf2 mouse and kidney from littermate control. (D) H\&E staining of Wt1-lgf2 mouse tumor and human WT. (E) Real-time RT-PCR expression quantification for 3 representative WT signature genes from 5 Wt1-lgf2 tumors (gray) and 5 E14.5 control kidneys (white). All 3 genes were upregulated in tumors $(P<0.05)$. Scale bars: $200 \mu \mathrm{m}$ (A); $1 \mathrm{~cm}$ (C); $300 \mu \mathrm{m}$ (D, top); $150 \mu \mathrm{m}$ (D, bottom).
$W t 1$-null mouse strain, $W t 1^{f l}$. Following Cre-mediated recombination of the $W t 1^{f l}$ allele, the resultant $W t 1^{\Delta}$ allele encodes a truncated mutant protein and is phenotypically indistinguishable from the $\mathrm{Wt} 1^{-}$allele (15). We introduced into $\mathrm{Wt} 1^{-/ f l}$ mice the $H 19^{-}$allele that results in expression of the normally silenced maternal copy of Igf2 (16) and Cre-ER ${ }^{T M}$, a tamoxifen-inducible (TM-inducible) Cre-expressing transgene (17). Because the degree of Cre function is depending on TM dose, we were able to ablate $W t 1$ somatically in a small subset of cells so that kidney development and animal viability were not compromised. With these tools, we successfully established the first mouse model to our knowledge for WT, by using gene mutations known to occur in human tumors. Using this model, we defined one combination of alterations that frequently results in tumors and identified the ERK signaling pathway as being dysregulated in mouse and human tumors.

Additionally, this model provides an example of how differences in chromosomal organization between humans and mice can affect the development of appropriate mouse models for human disease and how an awareness of these differences and subsequent careful experimental design can be key for generating such a model.

\section{Results}

Wt1-Igf2 mice develop tumors at high frequency. A tumor-watch cohort of 11 mutant mice ( $W t 1^{-/ f l} H 19^{+/-m} C r e-E R^{T M}$; referred to herein as Wt1-Igf2 mice) and 27 littermate controls (Wt1 $1^{+/ f l} \mathrm{H} 19^{+/-m} \mathrm{Cre}-E R^{T M}$, $W t 1^{-/ f l} H 19^{+/-m}$, and $\left.W t 1^{+/ f l} H 19^{+/-m}\right)$ were generated as described in Methods. Of note, all embryos carried a maternally inherited ${\mathrm{H} 19^{-}}^{-}$allele (H19-m) that results in upregulation of Igf2 as a result of expression of the normally silent maternal allele (16). Embryos were treated in utero at E11.5 with a TM dose of $1 \mathrm{mg} / 40 \mathrm{~g}$ body weight, which resulted in Cre-mediated recombination in approximately $5 \%-10 \%$ of kidney cells (Figure 1A) and no decreased viability of mutant embryos. Palpable tumors were noted in mutant mice beginning at 9 weeks of age (Figure 1B). At 19 weeks of age, all remaining mutant and control mice were sacrificed and assessed for tumor development. In total, tumors were present in 7 of $11(64 \%)$ mutants and 0 of 27 controls. Bilateral or multicentric tumors were observed in 6 of 7 animals. Tumors were usually large in dimension and weighed about 10 -fold more than the littermate control kidneys (Figure 1C). To study whether Wt1 inactivation by itself is sufficient for tumor development, a separate cohort of 23 similarly TM-treated $W t 1^{-/ f l} C r e-E R^{T M}$ mice, which did not carry the H19-m allele, was also generated. At 4-7 months of age, no tumors were observed in these mice. These data indicate that either Wt 1 ablation or Igf 2 upregulation alone is not sufficient for tumor development and suggest that they affect 2 different cellular processes whose dysregulation is critical for WT development. This finding is reminiscent of mouse models for pancreatic $\beta$ cell carcinoma and intestinal adenomas, in which Igf2 upregulation is associated with cellular hyperproliferation and plays a key role in tumorigenesis, but only in the context of activation of an oncogene or inactivation of a tumor suppressor gene $(18,19)$. 

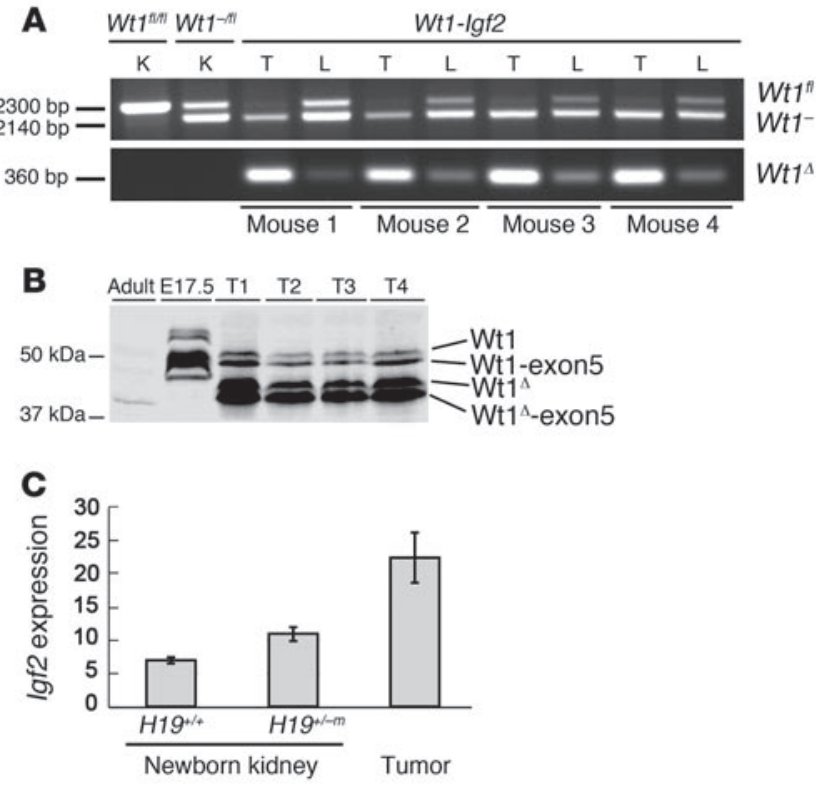

D Tumor-bearing kidney

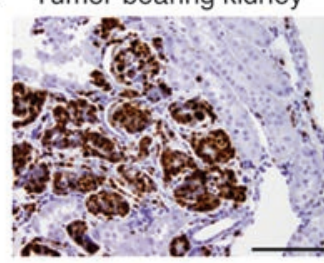

E
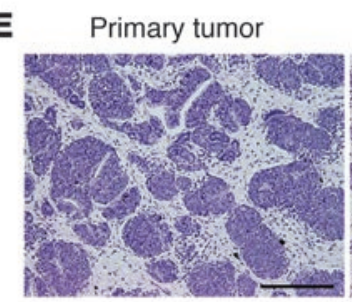

Control kidney

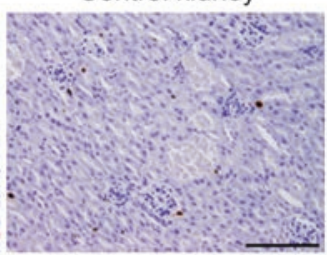

Xenograft

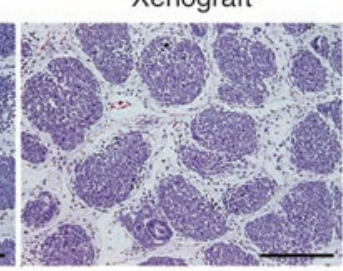

Figure 2

Wt1-lgf2 mouse tumors are clonal, hyperproliferative, and malignant. (A) PCR detection of $W t 1^{f l}$ and $W t 1^{\Delta}$ alleles in liver (L), and predominance $(\sim 90 \%)$ of $W t 1^{\Delta}$ allele in tumors (T), from 4 Wt1-Igf2 mice. (B) Western blot demonstrating predominant expression of the truncated WT1 protein encoded by the $W t 1^{\Delta}$ allele. Both wild-type and mutant proteins displayed isoforms resulting from the normal alternative splicing of $W t 1$ exon 5 . (C) Elevated Igf2 expression in tumors, quantified by real time RT-PCR. (D) Ki67 IHC of a section containing mouse WT and adjacent normal kidney and a kidney section from a littermate control demonstrating hyperproliferation in the tumor. (E) H\&E staining of a primary tumor and its xenograft tumor. Scale bars: $200 \mu \mathrm{m}$.

Wt1-Igf2 tumors recapitulate the histology and signature gene expression of human WTs, are derived from Wt1-ablated cells, and can be passaged in NOD/SCID mice. To determine whether the Wt1-Igf2 mouse is a relevant model for human WT, we first analyzed tumor tissues with $\mathrm{H} \& \mathrm{E}$ staining. Mouse tumors displayed a histology very similar to that of typical, triphasic WTs, with predominant blastemal and epithelial cells along with stromal elements (Figure 1D), although, as in human tumors, regions of tumors in which 1 or 2 elements predominated were also observed. Prior analysis of human tumors has identified a WT signature of genes overexpressed relative to fetal kidneys (20). Of particular interest, given the hypothesized role of aberrant developmental processes in WT, were 3 genes (Six1, Pax2, and Eya1) that are expressed in nephrogenic mesenchyme and play critical roles in kidney development (21-23). As was observed in human tumors, the Wt1-Igf2 mouse tumors exhibited upregulation of these genes (Figure 1E). In summary, these data indicate that Wt1-Igf2 tumors recapitulate the phenotype of human WT, both histologically and molecularly.

Genotypic and Western blot analyses demonstrated the predominant presence of the recombined $W t 1^{\Delta}$ allele and its encoded truncated protein (Figure 2, A and B). These data indicate that although the tumors are likely multifocal, given that $W_{t} 1^{f l}$ recombination was estimated to have occurred in approximately $5 \%-10 \%$ of cells, each focal tumor arose as a clonal expansion of a cell in which Wt1 was ablated. Moreover, tumors displayed elevated Igf2 expression (Figure 2C) and were highly proliferative, as assessed by Ki67 staining (Figure 2D). Injection of NOD/SCID mice with dissociated tumor cells cultured overnight resulted in xenograft tumors whose histology was very similar to that of the primary tumor (Figure 2E). Therefore, the mouse WTs not only are malig- nant, but also, at least in early passages, maintain the original histology through transplantation.

Widespread ablation of Wt1 blocks kidney development, but does not induce widespread apoptosis. Germline ablation of Wt1 results in apoptosis of urogenital ridge cells and renal/gonadal agenesis (10). However, the observation of homozygous WT1 mutations in tumors implies that later during kidney development, WT1 ablation results in tumorigenesis, not apoptosis. The histologic features of human WTs (reminiscent of differentiating renal mesenchyme that expresses WT1) suggest that in vivo WT1 ablation at later stages of kidney development impairs normal differentiation, leading to the accumulation of cells that, with appropriate additional molecular changes, can become malignant. Testing this model in vivo, however, has been problematic because of the renal agenesis resulting from germline WT1 ablation.

Accordingly, we determined the effect of somatic Wt1 ablation on differentiating mesenchyme. We treated $W t 1^{-f f l} C r e-E R^{T M}$ and littermate control embryos at E11.5 with a $3 \mathrm{mg} / 40 \mathrm{~g}$ TM dose, which resulted in Cre-mediated recombination in approximately $85 \%-90 \%$ cells of the kidney (Figure 3A). Mutant kidneys were examined at birth (E19) and displayed a complete block in nephron development; no glomeruli were present, and there was no differentiation of condensed mesenchyme past the comma-shaped body stage (Figure 3B). We further assessed the developmental effect of Wt1 ablation using in vitro culture of kidney rudiments from E12.5 embryos treated with $3 \mathrm{mg} / 40 \mathrm{~g}$ TM at E11.5. Mutant explants cultured for 3 days in medium with $1 \mu \mathrm{M}$ 4-hydroxyl TM (4-OHTM) exhibited approximately $95 \%$ downregulation of $W t 1$ expression by quantitative PCR (Wt1-/fl Cre-ER ${ }^{T M}$ mutant, $1.85 \pm 1.69$; $W t 1^{+/ f l} \mathrm{Cre}-E R^{T M}$ control, $\left.25.04 \pm 3.24 ; P=0.00001, n=4\right)$ and 
A

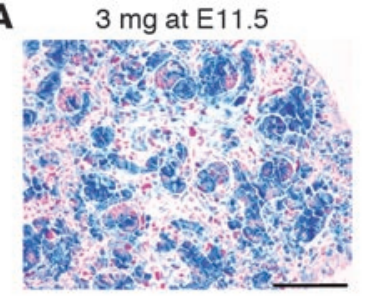

c
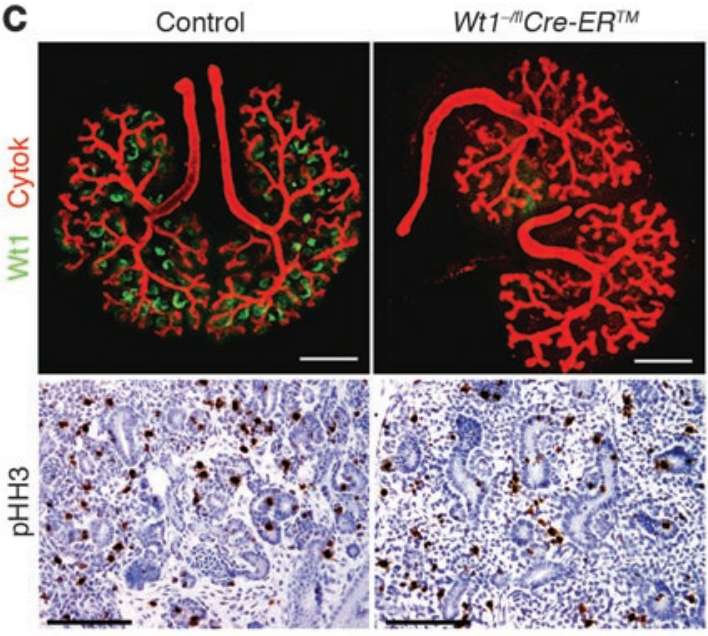

D

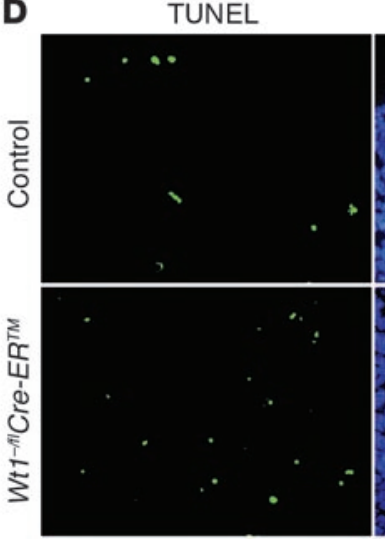

B

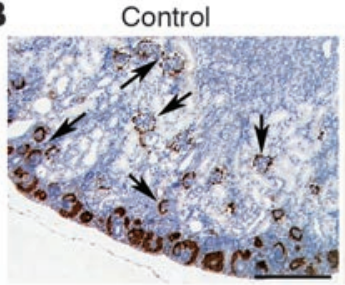

Wt1 ${ }^{-n I}$ Cre-ER ${ }^{T M}$

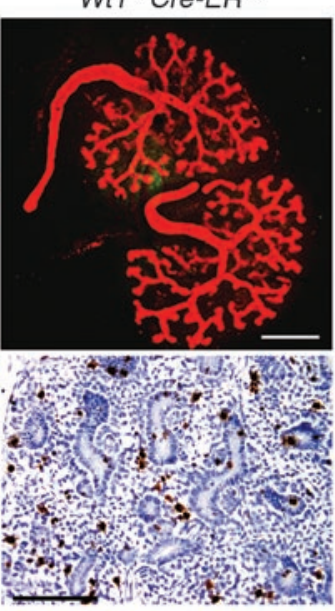

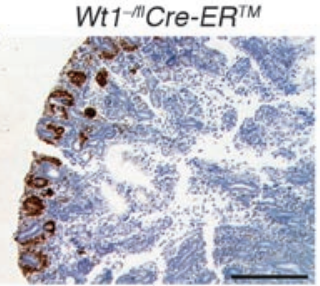

DAPI

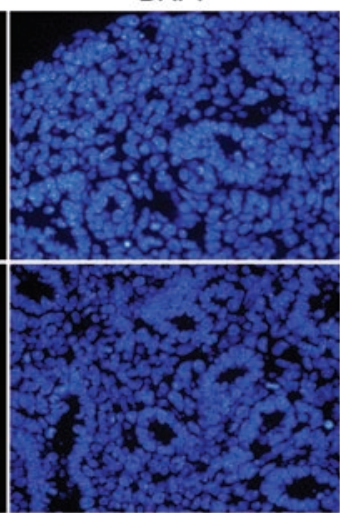

Merge

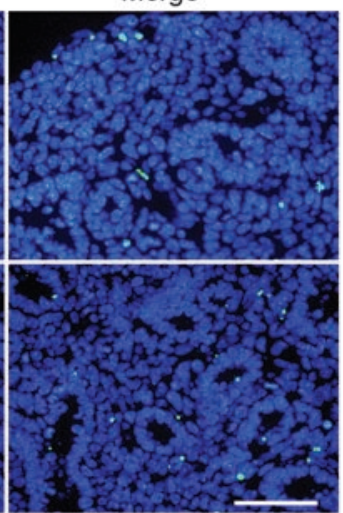

\section{Figure 3}

Wt1 ablation at approximately $\mathrm{E} 13$ results in a dramatic block in kidney development, but only a slight increase in apoptosis. (A) X-gal staining, showing Cre-mediated recombination estimated at approximately $85 \%-90 \%$, in E14.5 kidney cells following $3 \mathrm{mg} / 40 \mathrm{~g}$ tamoxifen at E11.5. (B) WT1 $\mathrm{IHC}$ of newborn kidneys from $W_{t 1^{-f I}} \mathrm{Cre}-E R^{T M}$ and littermate controls. Nephrogenic structures and mature glomeruli (arrows) were observed in control, but not mutant, kidney. WT1 antibody detects both wild-type and mutant protein. (C) Double immunofluorescence staining of whole-mount kidney explants cultured for 3 days, showing a block in nephrogenesis, but no effect on ureteric bud branching in the mutant. Green, WT1; red, cytokeratin. (D) TUNEL analysis revealed little apoptosis in E13.5 kidneys. Scale bars: $200 \mathrm{~mm}$ (A); 400 mm (B); 300 mm (C); $100 \mu \mathrm{m}$ (D). exhibited a similar complete block in condensed mesenchyme differentiation, as evidenced by the absence of comma-shaped bodies and more differentiated nephrogenic structures (Figure $3 \mathrm{C}$ ). These in vivo and ex vivo data are consistent with previously published observations following siRNA knockdown of Wt1 in kidney organ culture (24). The ex vivo data also clearly indicated that the bifurcation of the ureteric bud was not noticeably impaired in the mutant rudiment. While this could potentially be due to the residual approximately $5 \% \mathrm{Wt} 1$ expression observed in the cultured rudiments, these data suggest that mesenchyme in which $W t 1$ is somatically mutated retains its capacity to induce the branching of the ureteric bud. No increased cell proliferation was noted in mutant rudiments by phospho-Histone $\mathrm{H} 3$ (pHH3) IHC (Figure 3C), with 3.56\% \pm 0.78\% pHH3-positive cells in mutant kidneys compared with $3.69 \% \pm 0.94 \%$ pHH3-positive cells in control kidneys $(P=0.87, n=3)$.

To determine the more immediate effects of Wt 1 ablation on mesenchyme differentiation, we assessed mutant kidneys for apoptosis at E13.5 and E14.5, 2 time points at which histologic differences between mutant and control were minimal. Apoptosis was also assessed at a later time point (i.e., newborn). As shown by TUNEL analysis, apoptosis in control kidneys at all 3 time points was approximately $0.9 \%$. Wt 1 ablation resulted in an increase in apoptosis that, while less than doubled, was statistically significant (Table 1 and Figure 3D). Notably, this was observed in the absence of Igf2 upregulation, which suggests that, although upregulation of the IGF signaling pathway can have an antiapoptotic effect in cells (25), Wt1-ablated mesenchyme displays little increased apoptosis and does not require rescue by Igf 2 upregulation.

Genes expressed in nephrogenic, but not stromagenic, mesenchyme are downregulated following Wt1 ablation. Following induction by the ureteric bud, the metanephric mesenchyme gives rise to both the epithelial cells of the mature nephron and the stromal cells of the mature kidney. Genes specifically expressed in these differently fated subsets of mesenchyme have been identified, and we assessed the effect of Wt1 ablation on the expression of these 


\begin{tabular}{|c|c|c|c|}
\hline Age & Control & Wt1 ablated & $P$ \\
\hline E13.5 & $0.88 \% \pm 0.36 \%$ & $1.56 \% \pm 0.53 \%$ & 0.0496 \\
\hline E14.5 & $0.94 \% \pm 0.12 \%$ & $1.87 \% \pm 0.43 \%$ & 0.006 \\
\hline Newborn & $0.94 \% \pm 0.18 \%$ & $1.51 \% \pm 0.25 \%$ & 0.034 \\
\hline
\end{tabular}

Control, Wt1+/flCre-ER ${ }^{T M} ; W t 1$ ablated, Wt1-|fl$C r e-E R^{T M}$. Values are from 5 kidneys (4 largest sagittal sections per kidney) per time point and genotype.

genes in $\mathrm{E} 14.5 \mathrm{Wt1^{-/fl }}$ and $\mathrm{Wt} 1^{-/ f l} \mathrm{Cre}-E R^{T M}$ kidneys from embryos treated with $3 \mathrm{mg} / 40 \mathrm{~g}$ TM at E11.5. The expression of Pbx1, normally expressed in both uninduced mesenchyme and induced mesenchyme that differentiates into stroma (26), was unchanged in Wt1-ablated E14.5 kidneys (Table 2). Expression of Six2, a gene critical for the maintenance of a nephron progenitor population $(27,28)$; amphiregulin (Areg), whose expression pattern is similar to WT1 and which has been previously reported to be regulated by WT1 (29); and Foxd1, a marker for stromagenic mesenchyme $(30,31)$, were likewise unchanged following Wt1 ablation. Similarly, no significant change in E-cadherin (Chd1) expression was observed, although these data were confounded by the expression of this gene in the ureteric bud and its derivatives in addition to its upregulation in S-shaped bodies (32); changes in its expression in the differentiating mesenchyme was likely masked by its robust expression in the ureteric bud-derived components of the kidney. In contrast, mutant E14.5 kidneys displayed decreased expression of Sall1, normally expressed in uninduced mesenchyme and upregulated in induced mesenchyme, and $L h x 1$ and Wnt4, whose expression is detected only upon formation of pretubular aggregates (32-36). These data indicate that Wt1 ablation specifically affected the maturation of mesenchyme fated to become nephrons, but not the mesenchyme fated to become stroma.

To investigate the fate of the Wt1-ablated nephrogenic mesenchyme further, we assessed kidneys at E15.5, a time point at which all stages of nephron development are normally observed. Similar to what was observed in mutant newborn kidney and explant cultures, comma-shaped bodies and more mature nephrogenic structures were rarely present in mutant E15.5 kidneys (likely because of the lack of Cre recombinase activity observed in approximately $10 \%$ of mesenchyme), although nephrogenic mesenchyme was present, as detected by WT1 IHC (using antibody that detects the mutant protein) and SIX2 IHC (Figure 4). Of note, mesenchyme in the mutant was loosely condensed compared with the control. Moreover, mutant mesenchyme did not express K-cadherin (CDH6), an early marker of mesenchymal epithelialization expressed in renal vesicles, comma-shaped bodies, and the proximal end of S-shaped bodies (Figure 4 and refs. 37, 38). Similarly, mutant mesenchyme did not express E-cadherin (CDH1), which is expressed in the ureteric bud and the distal end - adjacent to the ureteric bud - of S-shaped bodies and subsequent structures (Figure 4 and ref. 38). Because the branching of the ureteric bud, and hence the morphogenesis of collecting ducts, appeared normal in the Wt1-ablated kidney (Figure 3), these structures were similarly stained with E-cadherin in both mutant and control kidneys; however, there was no evidence from E-cadherin IHC of early epithelial transformation of the mutant mesenchyme (Figure 4). Thus, both
K-cadherin and E-cadherin IHC demonstrated a lack of epithelial differentiation in the Wt1-ablated mesenchyme.

Upregulation of $P I R S$ and $p E R K 1 / 2$ effector pathway in tumors. IGF2 is a fetal mitogen that signals through the IGF-I receptor (IGF-IR; refs. 39-41). To identify the dysregulated transducing pathway(s) in tumors, we performed reverse-phase protein array (RPPA) analysis with 84 validated antibodies for components of various signaling pathways, including the IGF-IR, $W n t$, and TGF- $\beta$ pathways. Generally, little difference in protein expression was observed between the Wt1-Igf2 mouse tumors and kidneys from Wt1 $1^{-/ f l} H 19^{+/-m}$ newborns. However, this analysis did reveal that phosphorylation of IRS1, a substrate for IGF-IR, was increased in tumors relative to the newborn kidneys. More strikingly, phosphorylation of ERK1/2 in the Ras/Raf/MEK/ERK pathway downstream of IGF-IR signaling was strongly upregulated. In contrast, activation (i.e., phosphorylation) of alternative IGF-IR transducing pathway components AKT, phosphoinositide-dependent kinase 1 (PDK1), mammalian target of rapamycin (mTOR), p70 S6 kinase (70S6K), and STAT3 was not observed (Figure 5A). Western blot and IHC analyses confirmed that tumors had increased pERK1/2 levels (Figure 5, B and C).

RPPA analysis of human WTs similarly revealed a striking increase in pIRS and pERK1/2 in 18 of 26 of tumors, but little change in phosphorylation of AKT, PDK1, 70S6, or STAT3 relative to fetal kidney (Figure 5E). These data indicate that the dysregulated pathway identified in mouse tumors represents a relatively common tumorigenic mechanism in WTs that are genetically very heterogeneous. Consistent with the mouse tumor data, 5 human tumors (T2, T3, T7, T8, and T10; Figure 5E) that display both WT1 and IGF2 alterations all displayed elevated PIRS and pERK1/2, and T3 had the highest level of pERK1/2 of all the analyzed tumors.

Upregulation of $p E R K 1 / 2$ in fetal kidney as a result of Ig $f 2$ upregulation. pERK $1 / 2$ is normally expressed at low levels in developing kidney (42). To assess the effect of Wt1 ablation and/or Igf2 upregulation on pERK1/2 expression, we carried out IHC on E14.5 kidneys from embryos treated at E11.5 with $3 \mathrm{mg} / 40 \mathrm{~g}$ TM. In the absence of Igf2 upregulation (no $\mathrm{H}^{-m}{ }^{-m}$ allele), pERK1/2 was detectable in normal Wt1-fll control and Wt1-ablated kidneys, and no salient difference between these groups was observed (Figure 5D). With Igf2 upregulation (H19-m allele), pERK1/2 was strongly upregulated in both mesenchyme and ureteric bud, regardless of Wt1 ablation

\section{Table 2}

Real time RT-PCR quantification of gene expression in E14.5 kidneys following Wt1 ablation

\begin{tabular}{lrrc}
\hline Gene & \multicolumn{1}{c}{ Control } & Wt1 ablated & $\boldsymbol{P}$ \\
Wt1 & $17.15 \pm 2.17$ & $3.21 \pm 1.49$ & 0.00001 \\
Wnt4 & $2.06 \pm 0.13$ & $1.17 \pm 0.15$ & 0.000004 \\
Lhx1 & $2.11 \pm 0.40$ & $1.29 \pm 0.20$ & 0.003 \\
Sall1 & $1.43 \pm 0.03$ & $1.15 \pm 0.13$ & 0.005 \\
Pbx1 & $1.29 \pm 0.30$ & $1.60 \pm 0.29$ & 0.119 \\
Six2 & $1.08 \pm 0.06$ & $1.14 \pm 0.13$ & 0.412 \\
Foxd1 & $20.75 \pm 1.68$ & $15.45 \pm 5.48$ & 0.138 \\
Cdh1 & $5.95 \pm 0.99$ & $5.38 \pm 1.27$ & 0.453 \\
Areg & $2.68 \pm 0.66$ & $2.91 \pm 0.92$ & 0.645 \\
\hline
\end{tabular}

Control, $W t 1^{+/ f \mid} C r e-E R^{T M} ; W t 1$ ablated, $W t 1^{-|f|} C r e-E R^{T M}$. Values are for 5 pooled samples per genotype. Each pool consisted of kidneys from 3 embryos. 


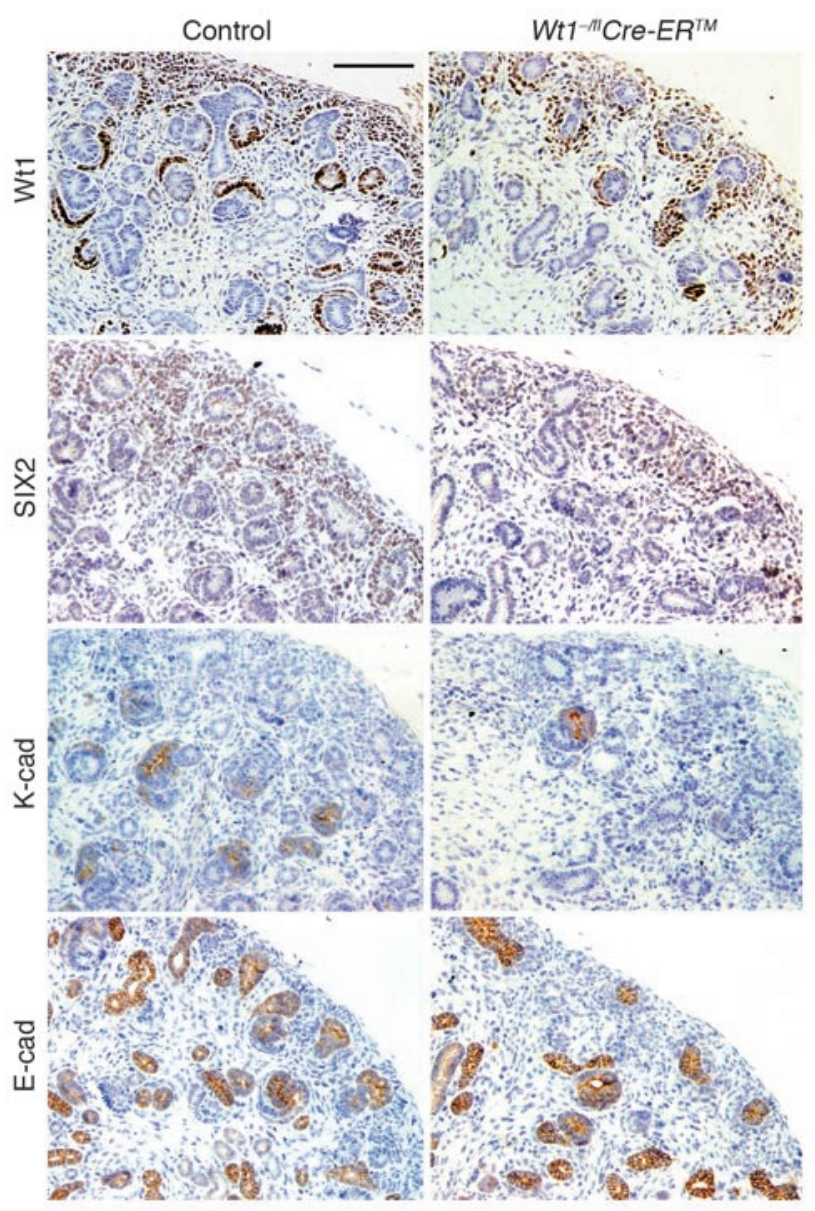

(Figure 5D). Given that WTs are thought to arise from mesenchyme, WT1 ablation was observed in human tumors, Wt1 ablation was required for tumorigenesis in this mouse model, and pERK1/2 was robustly expressed in these tumors, we hypothesize that the tumors arose from $W t 1$-ablated mesenchymal cells displaying aberrantly high pERK1/2 (Figure 5D, bottom right).

\section{Discussion}

Children heterozygous for a germline WT1 mutation are at a greatly elevated risk of developing WT, and tumors from these children have invariably mutated or lost the wild-type allele. In contrast, $W t 1^{+/-}$mice do not develop tumors. We hypothesized that this was due to the inability of differentiating murine metanephric mesenchyme to accrue necessary mutations during the developmental stage at which it was at risk for tumorigenesis. We further hypothesized that this inability was due in large part to differences in genomic organization between humans and mice - specifically, the synteny of WT1 and IGF2 in humans that is not present in mice. Thus, we needed to engineer into murine developing kidney cells 2 genetic alterations, somatic Wt1 homozygous ablation and Igf2 upregulation, that can result from a single event in humans. The early and frequent occurrence of tumors observed in the engineered Wt1-Igf2 mouse strongly supports our hypotheses and serves as an example of the need to be cognizant of genomic architecture when developing mouse models for human diseases.

The Wt1-Igf2 mouse also demonstrates that Wt1 ablation and Igf 2 upregulation constitute 1 combination of rate-limiting events

\section{Figure 4}

Lack of epithelialization of Wt1-mutant mesenchyme. IHC with WT1 and SIX2 antibodies demonstrated presence of mesenchymal cells in mutant kidneys (mutant, truncated WT1 protein is detected by WT1 antibody). Staining of mutant mesenchyme with antibodies to K-cadherin and E-cadherin, 2 markers of early epithelial differentiation, did not reveal any evidence of mesenchyme-to-epithelial transition in mutant mesenchyme, although ureteric buds and derivatives, which also express E-cadherin, were observed in mutant kidney. Scale bar: $200 \mu \mathrm{m}$.

for tumorigenesis and provides important insights into the cellular effect of these 2 alterations. The role of WT1 ablation vis-a-vis $\mathrm{LOH}$ or LOI at $11 \mathrm{p} 15$ imprinted genes (sometimes referred to as WT2), a result of which is biallelic expression of IGF2, has been unclear. These 2 events are observed in approximately $20 \%$ and $70 \%$ of human WT, respectively, which has led to speculation that they represent distinct molecular etiologies for WT. However, as in the Wt1-Igf2 mouse tumors, both WT1 ablation and 11p15 LOH/ LOI are present in some human tumors (14). The Wt1-Igf2 mouse data suggest a model, discussed below, in which upregulation of Igf2 results in increased ERK1/2 phosphorylation, Wt 1 ablation results in a block in mesenchyme differentiation, and the combined occurrence of these events leads to malignant tumors.

In addition to $11 \mathrm{p} 15 \mathrm{LOH} / \mathrm{LOI}$, a role for IGF2 in WT tumorigenesis is strongly suggested by the observation that patients with the somatic overgrowth syndrome Beckwith-Wiedemann syndrome who develop WT often carry germline mutations in an IGF2 imprinting control region (43, 44). IGF2 signals primarily through IGF-IR (39-41), and the increased expression of pIRS, a substrate for activated IGF-IR, in mouse tumors confirms that this is also true for the Wt1-Igf2 tumors. However, the IGF-IR signal can be transduced via several alterative signaling pathways, and to our knowledge, the effector signaling pathway key for IGF2's role in WT has not previously been known. The robust upregulation of pERK1/2 and lack of increased pAKT, pPDK1, pmTOR, and pSTAT3 in tumors indicates that in the Wt1-Igf2 mouse tumors, the IGF2 signal is transduced via the Ras/Raf/MEK/ERK pathway, which was not to our knowledge previously implicated in WT.

We determined that human WTs similarly display little evidence of activation of PDK1, STAT3, or AKT. In contrast, as in the mouse tumors, upregulation of pIRS1 and pERK1/2 was observed in many human WTs, confirming that the signaling pathway identified in the mouse model has indeed identified a pathway important in human tumors. That this pathway is not upregulated in all human tumors is likely in part a consequence of the known genetic heterogeneity of human WTs along with their more complex genetic background.

We detected no mutations in Ctnnb1, Wtx, and $p 53$, genes mutated in $5 \%-20 \%$ of human tumors, in the mouse tumors (data not shown). Similarly, genome-wide comparative genome hybridization (CGH) analysis revealed no duplication and/or loss of large genomic regions (e.g., 16q, 12q, 1p) that are sometimes observed in human tumors (data not shown). Although we cannot rule out the presence of mutations at other, previously unimplicated, loci in the mouse tumors, these data suggest that Wt1 ablation and Igf2 upregulation are the critical genetic events in these tumors.

WT1 was originally identified by virtue of its role as a tumor suppressor gene; roughly $20 \%$ of WTs are homozygous for WT1 lossof-function mutations. Interestingly, and seemingly contradictory to its role as a tumor suppressor gene, in mice, germline ablation 
A

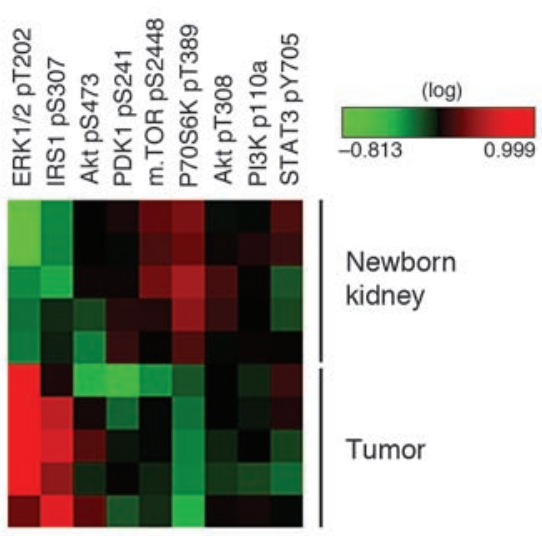

B

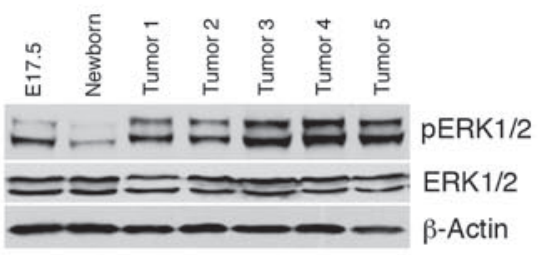

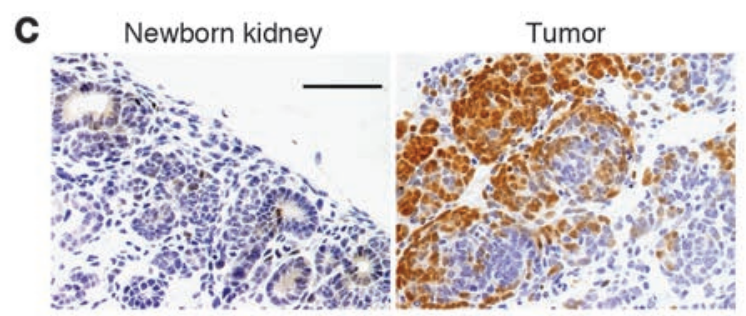

D

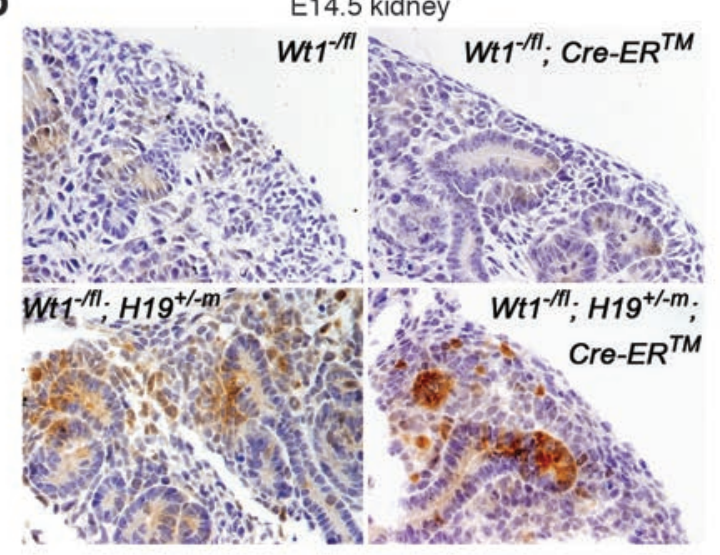

E
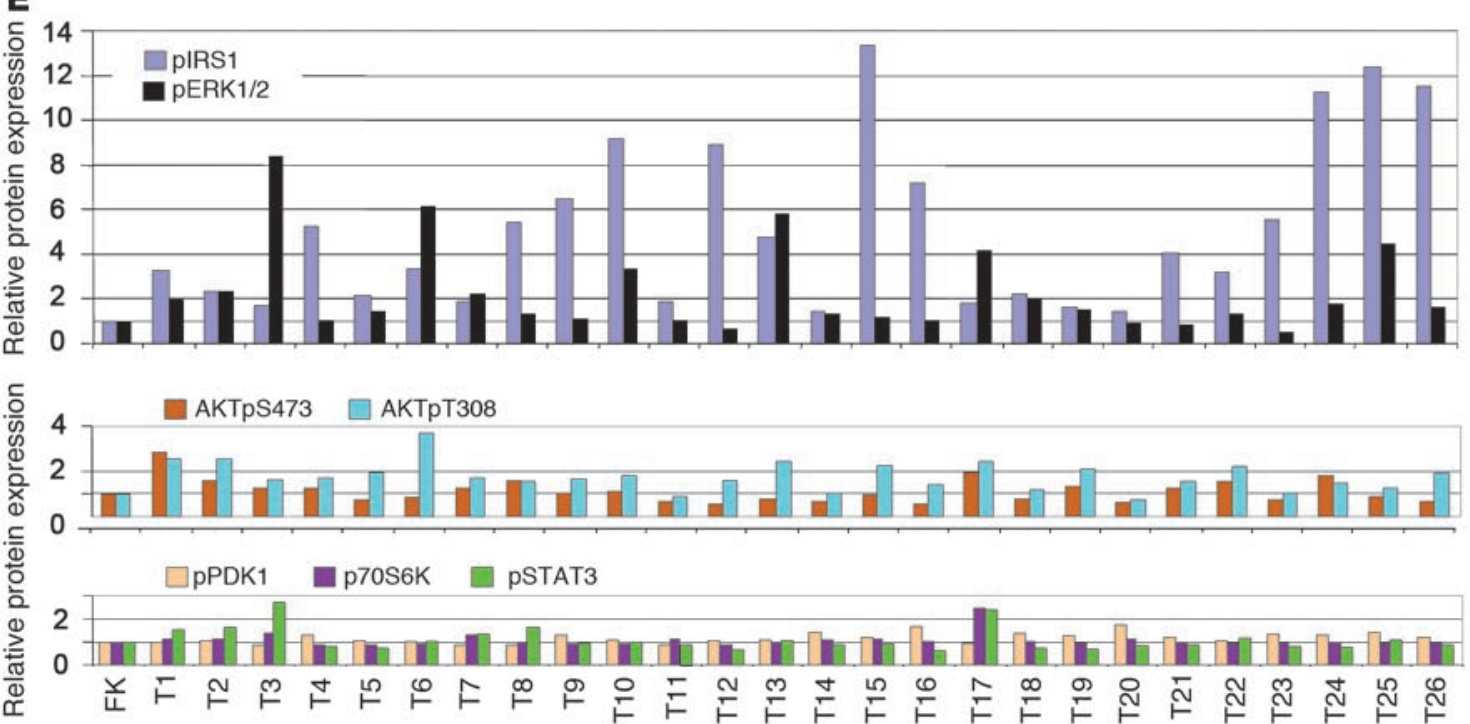

Figure 5

Increased pERK1/2 and pIRS1 expression in Wt1-lgf2 tumors and human tumors. (A) Heatmap of RPPA protein expression data in Wt1-lgf2

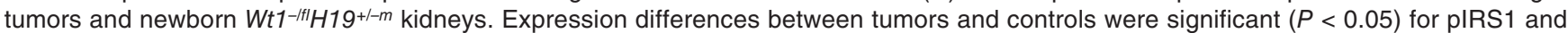
pERK1/2, but not for pPDK1 or pAKT. (B) Western blot analysis of $W t 1^{-/ f t} H 19^{+/-m}$ kidneys and tumors. (C and D) pERK1/2 IHC of newborn and

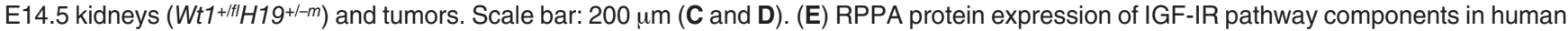
WTs $(\mathrm{T})$. Expression level in fetal kidney (FK) was designated as 1.

of Wt1 leads to apoptosis of urogenital ridge cells at approximately E10.5, resulting in complete kidney agenesis. However, while we observed a slight increase in the number of TUNEL-positive cells in Wt1-ablated kidney, there was no widespread apoptosis of metanephric mesenchyme following Wt1 ablation at approximately E13. This result indicates that loss of $W t 1$ function has different effects depending upon developmental stage: before invasion of the intermediate mesenchyme by the ureteric bud, Wt 1 is essential for cell survival, but its loss in approximately E13 metanephric mesenchyme, following ureteric bud invasion, has only a weak apoptotic effect. The complete block in kidney development we observed histologically in vivo following genetic Wt1 ablation (which was previously described in vitro following Wt1 knockdown; ref. 24) is consistent with this notion.

While Wt1 is expressed in the metanephric mesenchyme from which both the nephrons and the kidney stroma arise, its expres- 
sion is increasingly restricted during nephrogenesis, such that its expression in adult kidney is primarily in podocytes, highly specialized epithelial cells in the glomerulus. Interestingly, and in contrast to a prior observation following in vitro knockdown of Wt1 in approximately E13 kidney (24), we found no increase in cell proliferation following $W t 1$ ablation. However, our analysis of Wt1ablated E14.5 kidneys revealed that, while little histologic change was apparent compared with control kidneys at that time point, there was already significant alteration in the expression of genes (e.g., Wnt4, Sall1, and Lhx1) upregulated during mesenchymal epithelialization and differentiation to form nephrons. The most statistically significant dysregulated gene, Wnt4, is expressed in both ureteric bud and metanephric mesenchyme derivatives, and it is critical for the mesenchyme-to-epithelial transition that occurs during nephron development $(32,36,45)$. While the decrease in Wnt4 expression in Wt1-ablated E14.5 kidneys was less than 2-fold, this change was nevertheless highly statistically significant. The somewhat modest change in Wnt4 expression is likely due to its normal expression in the ureteric bud derivatives; while Wt1 ablation affects Wnt4 expression in the mesenchyme, it cannot do so in ureteric bud derivatives that do not express Wt1. Wnt $4^{-/-}$mutant kidneys display a developmental phenotype similar to that of Wt1ablated embryonic kidneys (45), and in vitro studies have suggested that Wt1 indirectly upregulates Wnt4 expression (46). Our in vivo data strongly support this.

In contrast to Wnt4, Sall1, and $L h x 1$, changes in the expression of $P b x 1$ and Foxd1, expressed in mesenchyme fated to become kidney stroma $(26,30,31)$, was not observed. Interestingly, there was also no change in expression of Six2, a gene required for the maintenance of mesenchymal tubule progenitor cells $(27,28)$. These in vivo histology and gene expression data suggest a model whereby Wt1 ablation has little, if any, effect on stromagenic mesenchyme. Neither does it result in widespread loss of SIX2-positive nephron progenitors. Instead, it blocks - perhaps via loss of Wnt4 expression - the ability of induced mesenchyme to form renal vesicles. Mutant mesenchyme did not express K-cadherin, an early marker of epithelialization, but whether this is a primary consequence of Wt1 ablation or is secondary to the lack of renal vesicle initiation is not clear. The observation of epithelial differentiation in some components of both murine and human WTs implies that Wt1ablated, blocked mesenchyme can, in the context of other genetic alterations and/or cellular milieu, undergo epithelial differentiation, albeit aberrantly. Whether Igf2 upregulation plays a role in this regard is not known and requires further investigation.

From which mesenchymal cells did the Wt1-Igf2 tumors arise? The triphasic histology of stroma, blastema (similar to condensed mesenchyme), and epithelial tubules observed in both human and mouse tumors points to an origin in an early mesenchyme progenitor cell that then differentiates aberrantly. In this model, the expression in tumors of genes (Six1, Pax2, and Eya1) normally expressed in the nephrogenic mesenchyme could be a reflection of the impaired differentiation of the nephrogenic elements of the tumor. Alternatively, this nephrogenic gene expression profile could be a reflection of the tumor arising at this developmental stage, with epithelial and stromal components observed in the tumor arising as a result of aberrant differentiation and/or transdifferentiation. Additionally, even though Wt1 ablation did not alter the expression of markers for stromagenic mesenchyme or tubule progenitor cells, it is possible that Wt1 loss is tumorigenic in one or both of these cell types. Substitution of the ubiquitous
Cre transgene with kidney compartment-specific Cre transgenes in the Wt1-Igf2 mouse model will be one robust approach to investigate these alternative models of tumorigenesis.

In summary, we have generated an endogenous mouse model for WT by engineering into mice 2 separate genetic alterations that commonly occur in humans as a result of a single genomic event. In doing so, we have defined one important combination of genetic alterations that act as the requisite rate-limiting 2 hits originally hypothesized for WT tumorigenesis (47). Data from the Wt1-Igf2 mouse tumors and data from fetal kidneys that have sustained these 2 alterations individually suggest a model whereby loss of Wt1 function alters normal differentiation of the induced nephrogenic mesenchyme and $\operatorname{Ig} f 2$ upregulation drives the proliferation of these abnormal cells through IGF-IR signaling transduced via pIRS1 and pERK1/2. A significant fraction of human WTs also displayed increased pIRS1 and pERK1/2. Thus, guided by the mouse tumors, we have identified a signaling pathway that likely plays an important role in the development of many human WTs. Because the Wt1-Igf2 mice carry the same alterations that occur in human tumors, they provide a highly relevant model for further investigating the underlying biology of tumor development, for understanding the functional significance of other gene mutations previously identified in subsets of human WTs, and for functionally identifying additional genes that have been implicated in WT by linkage, LOH, CGH, and other studies. Furthermore, the IGF pathway has become increasingly recognized as an attractive target for cancer therapy (25), and the Wt1-Igf 2 animals potentially provide a powerful model for assessing the short- and long-term efficacy of anti-IGF pathway molecules for cancer treatment and perhaps also prevention.

\section{Methods}

Widespread or mosaic somatic inactivation of Wt1 and generation of tumor-watch cohort. All animal work was reviewed and approved by the IACUC of M.D. Anderson Cancer Center (Houston, Texas, USA). Genotyping of the mouse strains has been described previously $(15-17)$. Wt1 was inactivated mosaically or almost completely in $W t 1^{-/ f l} C r e-E R^{T M}$ embryos by in utero treatment of pregnant mice with TM ( 1 or $3 \mathrm{mg} / 40 \mathrm{~g}$ body weight) at E11.5. These treatments resulted in Cre-recombinase activity in approximately $5 \%-10 \%$ or approximately $85 \%$ of cells, respectively, and were used for tumor analysis and kidney development studies, respectively. To generate our mutant, tumor-watch cohort (Wt1 $\left.1^{-f l} H 19^{+/-m} \mathrm{Cre}-E R^{T M}\right)$ and littermate controls $\left(\mathrm{Wt} 1^{+/ f l} \mathrm{H} 19^{+/-m} \mathrm{Cre}-E R^{T M}, \mathrm{Wt} 1^{-/ f l} \mathrm{H} 19^{+/-m}\right.$, and $\left.\mathrm{Wt} 1^{+/ f l} \mathrm{H} 19^{+/-m}\right)$, we crossed $\mathrm{Wt}^{\mathrm{fl} / \mathrm{fl} l} \mathrm{H} 19^{-/-}$females with $\mathrm{Wt} 1^{+/-} \mathrm{Cre}-\mathrm{ER} \mathrm{R}^{\mathrm{TM}}$ males. The H19- mouse strain was provided by S. Tilghman (Princeton University, Princeton, New Jersey, USA). Of note, all embryos carried a maternally inherited $\mathrm{H}^{-{ }^{-}}$allele that results in upregulation of Igf2 as a result of LOI and biallelic expression of Igf2 (16). Cohorts of 11 mutant and 27 littermate controls, treated with $1 \mathrm{mg} / 40 \mathrm{~g}$ TM at E11.5, were generated along with an additional cohort of Wt1-fflCre-ER $R^{T M}$ animals similarly treated with $1 \mathrm{mg} / 40 \mathrm{~g}$ TM. This latter cohort enabled us to assess the effect of somatic, mosaic Wt1 ablation in the absence of Igf2 upregulation.

Generation of mouse WT xenografts. Primary tumors were dissociated by mincing and collagenase treatment, filtered through a $40-\mu \mathrm{m}$ strainer, cultured overnight, and injected i.p. $\left(2 \times 10^{6}\right.$ cells $)$ or s.c. $\left(5 \times 10^{6}\right.$ cells $)$ into NOD/SCID mice (Jackson Laboratory, JAX Stock 5557).

$R T-P C R$. RNA was isolated from snap-frozen tissues using RNAqueous4PCR kit (Ambion), and gene expression was quantified by SYBR Green assays (Applied Biosystems). Equal amounts of total RNA were mixed with MultiScribe Reverse Transcriptase (Applied Biosystems) and PCR Master Mix. First-strand cDNA was generated at $48^{\circ} \mathrm{C}$ for 30 minutes and subse- 
quently amplified using ABI 7900 HT sequence detection system (Applied Biosystems). Data were analyzed by the comparative $\mathrm{Ct}$ method relative to Gapdh as an endogenous control (48) and expressed as fold differences to a common reference value, which had the highest relative $\mathrm{Ct}(\Delta \mathrm{Ct})$ and whose expression level was designated as 1 . Data from 5 pools of kidney for each genotype were statistically assessed with 2 -tailed Student's $t$ test. RT-PCR primer sequences were: Areg forward, 5'-GCTGTTGCTGCTGGTCTTAGG-3'; Areg reverse, 5'-GGGTCATTGAGCTCCAAAGC-3'; Cdh1 forward, 5'-CTGTGGACGTGGTAGACGTGAA-3'; Cdh1 reverse, 5'-CGAGCGGTATAAGATGTGATTTCC-3'; Eya1 forward, 5'-CACCCAGACAAGGTTCATTGC-3'; Eya1 reverse, 5'-TCCCATCACCCTCTAGGTTTATG-3'; Foxd1 forward, 5'-TTCCCCGCTTGGCAGAA-3'; Foxd1 reverse, $5^{\prime}$-TCTTGACGAAGCAGTCGTTGA-3'; Gapdh forward, 5'-GCAGTGGCAAAGTGGAGATTG-3'; Gapdh reverse, 5'-AATTTGCCGTGAGTGGAGTCA-3'; Igf2 forward, 5'-AGAGAGGCCAAACGTCATCGT-3'; Igf2 reverse, 5'-TCACCCCACCTTGCAGAATTA-3'; $L h x 1$ forward, 5'-CAACCTGACCGAGAAGTGCTT-3'; $L h x 1$ reverse, 5'-CGCATTTGGTACCGAAACATC-3'; Pax2 forward, 5'-GATCGAGTCTTTGAGCGTCCTT-3'; Pax2 reverse, 5'-GAGAGTATTCATTCCCCTGTTCTGA-3'; $P b x 1$ forward, 5'-ACTCGCCCTCTACTCCCAACTC-3'; Pbx1 reverse, 5'-GAGTGACTGCACGCTCATGAAC-3'; Sall1 forward, 5'-AGAGAAGCCCTTTGCTTGCA-3'; Sall1 reverse, 5'-GTGCCCATGTGGACCTTCA-3'; Six 1 forward, 5'-GCAGCCCGGTGTTTGTTACTA-3'; Six 1 reverse, 5'-CTCCTGCTGGGCTACTGAGTTT-3'; Six2 forward, 5'-CTTGCTTTCCGTGCTTGATG-3'; Six2 reverse, 5'-TTCCAGTACGCGGCACAGA-3'; Wnt4 forward, 5'-CCGGGCACTCATGAATCTTC-3'; Wnt4 reverse, 5'-CACCCGCATGTGTGTCAAG-3'; Wt1 forward, 5'-CAAGGACTGCGAGAGAAGGTTT-3'; Wt1 reverse, 5'-TGGTGTGGGTCTTCAGATGGT-3'.

Histologic, IHC, and Western blot analysis. Paraformaldehyde-fixed, paraffin-embedded tissues were assessed by $\mathrm{H} \& \mathrm{E}$ or IHC as previously described (15). Western blots were performed as previously described (49) and imaged on a Li-Cor Odyssey Imager. Antibodies used were against WT1 (Santa Cruz Biotechnology Inc., sc-192), which recognizes an epitope present on the truncated protein encoded by $W t 1^{\Delta}$ allele; Ki67 (Abcam, ab15580); pHH3 (Upstate, 06-570); ERK1/2 (Cell Signaling Technology, 4377); pERK1/2 (Cell Signaling Technology, 4376); E-cadherin (Cell Signaling Technology, 3195); K-cadherin (gift from G. Dressler, University of Michigan, Ann Arbor, Michigan, USA; also Abcam, ab79005); and Six2 (Santa Cruz Biotechnology Inc.).

$X$-gal staining of kidney and estimation of Cre-mediated recombination. ROSA26R females were crossed with $C r e-E R^{T M}$ males, and the embryos were treated at E11.5 with TM at a dose of 1 or $3 \mathrm{mg} / 40 \mathrm{~g}$ maternal weight. Kidneys were dissected from embryos at E14.5, washed in PBS, and fixed for 30 minutes with a solution of $0.2 \%$ glutaraldehyde, $2 \%$ formalin, $5 \mathrm{mM}$ EGTA, and $2 \mathrm{mM} \mathrm{MgCl} 2$ in $0.1 \mathrm{M}$ phosphate buffer, $\mathrm{pH}$ 7.3. After washing 3 times in a rinse solution containing $0.1 \%$ sodium deoxycholate, $0.2 \% \mathrm{NP} 40,2 \mathrm{mM}$ $\mathrm{MgCl}_{2}$, and $0.1 \mathrm{M}$ phosphate buffer, $\mathrm{pH} 7.3$, the kidneys were stained for $2-4$ hours with a staining solution consisting of $1 \mathrm{mg} / \mathrm{ml} \mathrm{X-gal,} 5 \mathrm{mM}$ potassium ferricyanide, $5 \mathrm{mM}$ potassium ferrocyanide, and a rinse solution. The kidneys were postfixed in $4 \%$ paraformaldehyde and embedded in paraffin. Sections from the kidneys were counterstained with eosin and used to estimate Cre-mediated recombination.

Metanephric kidney explant culture. Embryos from the cross of $W t 1^{f / / l}$ females with $\mathrm{Wt} t 1^{+/-} \mathrm{Cre}-\mathrm{ER} \mathrm{R}^{\mathrm{TM}}$ males were treated in utero at E11.5 with $3 \mathrm{mg} / 40 \mathrm{~g}$ TM via an i.p. injection of the pregnant female. Metanephric kidney rudiments were dissected from E12.5 embryos and cultured as previously described (50). Explants were cultured in the presence of $1 \mu \mathrm{M}$ 4-OH-TM (Sigma-Aldrich).
After culture, explants were fixed either in methanol for immunostaining or in $4 \%$ paraformaldehyde for immunohistochemistry. The explants were also snap frozen for RNA isolation. Immunostaining experiments with murine anti-cytokeratin (Sigma-Aldrich) and rabbit anti-WT1 (Santa Cruz Biotechnology Inc.) were performed similarly to the procedures described previously (50). Images were captured on a Zeiss LSM 510 confocal microscope.

Proliferation quantification. To quantify proliferation, IHC with kidney sections and antibodies to $\mathrm{pHH} 3$ was performed as described above. $\mathrm{pHH} 3$ positive cells, excluding those in ureteric buds, were counted against the number of total cells in the examined area. For each kidney, about 4,000 cells in total were counted; proliferation was expressed as a percentage of pHH3-positive cells. For each genotype, 3 kidneys were assessed.

TUNEL assay. TUNEL assays for TM-treated E13.5, E14.5, and newborn kidneys were carried out with the In Situ Cell Death Detection Kit, Fluorescein (Roche Applied Sciences), as recommended. For each time point and genotype, 5 kidneys (4 largest sagittal sections per kidney) were used. Images were acquired with a Leica DMR Epifluorescent microscope and MetaMorph software (Universal Imaging). Data collected were statistically analyzed with 2-tailed Student's $t$ test. Apoptosis was expressed as a percentage of TUNEL-positive cells relative to total DAPI-positive cells.

$R P P A$. Phosphorylated and unphosphorylated components of cell signaling pathways were quantified by RPPA as previously described (51). Briefly, protein extracts were prepared from mouse newborn kidney and from primary murine and human WTs. Human fetal kidney lysate was purchased (Abcam). Following quantification, extracts were denatured and diluted in 52 -fold serial dilutions, which were then arrayed on multiple slides along with positive and negative controls prepared from mixed cell lysates or dilution buffer. Each slide was probed with a validated primary antibody with respect to specificity, reproducibility, high dynamic range of the assay, correlation with Western blotting data, etc., and with a biotin-conjugated secondary antibody. After staining, slides were scanned, and spot intensities were analyzed, quantified, and normalized. $\log _{2}$ median centered data were used to generate heatmaps using the Cluster algorithm (52). Statistical analysis was done similarly as for RT-PCR data. For human tumors, linear RPPA data standardized to fetal kidney are presented.

Statistics. Significance of differences between samples from 2 groups was determined by 2 -tailed Student's $t$ test. Statistical differences were considered significant for $P$ values less than 0.05 . Statistical data were presented as mean \pm SD. Sample size was $n=5$ unless otherwise indicated.

\section{Acknowledgments}

We thank S. Tilghman for the gift of the H19- mouse strain, G. Dressler for supplying the K-cadherin antibody, Y. Lu for RPPA analyses, and D. Fang for Western blot assistance. This work was supported by NIH grants CA34936, DK069599, HD30284, NCI CCSG grant CA16672, and NCI training grant CA009299 and by the Odyssey Program and the Theodore N. Law Award for Scientific Achievement.

Received for publication May 20, 2010, and accepted in revised form October 6, 2010.

Address correspondence to: Vicki Huff, Department of Genetics, Unit 1010, University of Texas M.D. Anderson Cancer Center, 1515 Holcombe Blvd., Houston, Texas 77030, USA. Phone: 713.834.6384; Fax: 713.834.6380; E-mail: vhuff@mdacc.tmc.edu.
1. Bardeesy N, et al. Anaplastic Wilms' tumour, a subtype displaying poor prognosis, harbours p53 gene mutations. Nat Genet. 1994;7(1):91-97.

2. Huff V. Wilms tumor genetics. Am J Med Genet.
1998;79(4):260-267.

3. Koesters R, et al. Mutational activation of the betacatenin proto-oncogene is a common event in the development of Wilms' tumors. Cancer Res. 1999;
59(16):3880-3882.

4. Maiti S, Alam R, Amos CI, Huff V. Frequent association of beta-catenin and WT1 mutations in Wilms tumors. Cancer Res. 2000;60(22):6288-6292. 
5. Rivera MN, et al. An X chromosome gene, WTX, is commonly inactivated in Wilms tumor. Science. 2007;315(5812):642-645

6. Ruteshouser EC, Robinson SM, HuffV. Wilms tumor genetics: mutations in WT1, WTX, and CTNNB1 account for only about one-third of tumors. Genes Chromosomes Cancer. 2008;47(6):461-470.

7. Steenman MJ, Rainier S, Dobry CJ, Grundy P, Horon IL, Feinberg AP. Loss of imprinting of IGF2 is linked to reduced expression and abnormal methylation of H19 in Wilms' tumour. Nat Genet. 1994;7(3):433-439.

8. Ogawa $\mathrm{O}$, et al. Relaxation of insulin-like growth factor II gene imprinting implicated in Wilms' tumour. Nature. 1993;362(6422):749-751.

9. Park S, et al. Inactivation of WT1 in nephrogenic rests, genetic precursors to Wilms' tumour. Nat Genet. 1993;5(4):363-367.

10. Kreidberg JA, et al. WT- 1 is required for early kidney development. Cell. 1993;74(4):679-691.

11. Herzer U, Crocoll A, Barton D, Howells N, Englert C. The Wilms tumor suppressor gene Wt1 is required for development of the spleen. Curr Biol. 1999; 9(15):837-840.

12. Dao DD, et al. Genetic mechanisms of tumor-specific loss of 11p DNA sequences in Wilms tumor. Am J Hum Genet. 1987;41(2):202-217.

13. Schroeder WT, et al. Nonrandom loss of maternal chromosome 11 alleles in Wilms tumors. Am J Hum Genet. 1987;40(5):413-420.

14. Haruta M, et al. Duplication of paternal IGF2 or loss of maternal IGF2 imprinting occurs in half of Wilms tumors with various structural WT1 abnormalities. Genes Chromosomes Cancer. 2008;47(8):712-727.

15. Gao F, et al. The Wilms tumor gene, Wt1, is required for Sox9 expression and maintenance of tubular architecture in the developing testis. Proc Natl Acad SciU S A. 2006;103(32):11987-11992.

16. Leighton PA, Ingram RS, Eggenschwiler J, Efstratiadis A, Tilghman SM. Disruption of imprinting caused by deletion of the $\mathrm{H} 19$ gene region in mice. Nature. 1995;375(6526):34-39.

17. Hayashi S, McMahon AP. Efficient recombination in diverse tissues by a tamoxifen-inducible form of Cre: a tool for temporally regulated gene activation/inactivation in the mouse. Dev Biol. 2002; 244(2):305-318.

18. Christofori G, Naik P, Hanahan D. A second signal supplied by insulin-like growth factor II in oncogene-induced tumorigenesis. Nat Genet. 1995; 10(2):196-201.

19. Kaneda A, et al. Enhanced sensitivity to IGF-II signaling links loss of imprinting of IGF2 to increased cell proliferation and tumor risk. Proc Natl Acad Sci US A. 2007;104(52):20926-20931.

20. Li CM, et al. Gene expression in Wilms' tumor mimics the earliest committed stage in the metanephric mesenchymal-epithelial transition. Am J Pathol. 2002;160(6):2181-2190.

21. Torres M, Gómez-Pardo E, Dressler GR, Gruss P. Pax-2 controls multiple steps of urogenital devel- opment. Development. 1995;121(12):4057-4065

22. Xu PX, Adams J, Peters H, Brown MC, Heaney S, Maas R. Eya1-deficient mice lack ears and kidneys and show abnormal apoptosis of organ primordia. Nat Genet. 1999;23(1):113-117.

23. Xu PX, Zheng W, Huang L, Maire P, Laclef C, Silvius $\mathrm{D}$. Six 1 is required for the early organogenesis of mammalian kidney. Development. 2003; 130(14):3085-3094.

24. Davies JA, et al. Development of an siRNA-based method for repressing specific genes in renal organ culture and its use to show that the Wt1 tumour suppressor is required for nephron differentiation. Hum Mol Genet. 2004;13(2):235-246.

25 . Yuen JS, Macaulay VM. Targeting the type 1 insulinlike growth factor receptor as a treatment for cancer. Expert Opin Ther Targets. 2008;12(5):589-603.

26. Schnabel CA, Godin RE, Cleary ML. Pbx1 regulates nephrogenesis and Ureteric branching in the developing kidney. Dev Biol. 2003;254(2):262-276.

27. Self M, et al. Six2 is required for suppression of nephrogenesis and progenitor renewal in the developing kidney. EMBO J. 2006;25(21):5214-5228.

28. Kobayashi A, et al. Six 2 defines and regulates a multipotent self-renewing nephron progenitor population throughout mammalian kidney development. Cell Stem Cell. 2008;3(2):169-181.

29. Lee SB, et al. The Wilms tumor suppressor WT1 encodes a transcriptional activator of amphiregulin. Cell. 1999;98(5):663-673.

30. Hatini V, Huh SO, Herzlinger D, Soares VC, Lai E. Essential role of stromal mesenchyme in kidney morphogenesis revealed by targeted disruption of Winged Helix transcription factor BF-2. Genes Dev. 1996;10(12):1467-1478

31. Levinson RS, Batourina E, Choi C, Vorontchikhina M, Kitajewski J, Mendelsohn CL. Foxd1-dependent signals control cellularity in the renal capsule, a structure required for normal renal development. Development. 2005;132(3):529-539.

32. Brunskill EW, et al. Atlas of gene expression in the developing kidney at microanatomic resolution. Dev Cell. 2008;15(5):781-791.

33. Kobayashi A, Kwan KM, Carroll TJ, McMahon AP, Mendelsohn CL, Behringer RR. Distinct and sequential tissue-specific activities of the LIM-class homeobox gene Lim 1 for tubular morphogenesis during kidney development. Development. 2005; 132(12):2809-2823

34. Dressler GR, Deutsch U, Chowdhury K, Nornes HO, Gruss P. Pax2, a new murine paired-box-containing gene and its expression in the developing excretory system. Development. 1990;109(4):787-795

35. Takasato $M$, et al. Identification of kidney mesenchymal genes by a combination of microarray analysis and Sall1-GFP knockin mice. Mech Dev. 2004; 121(6):547-557.

36. Dudley AT, Lyons KM, Robertson EJ. A requirement for bone morphogenetic protein-7 during development of the mammalian kidney and eye. Genes Dev. 1995;9(22):2795-807.
37. Cho EA, Patterson LT, Brookhiser WT, Mah S, Kintner C, Dressler GR. Differential expression and function of cadherin- 6 during renal epithelium development. Development. 1998;125(5):803-812.

38. Shimazui $\mathrm{T}$, et al. Alterations in expression of cadherin- 6 and E-cadherin during kidney development and in renal cell carcinoma. Eur Urol. 2000; 38(3):331-338.

39. Kooijman R. Regulation of apoptosis by insulinlike growth factor (IGF)-I. Cytokine Growth Factor Rev. 2006;17(4):305-323.

40. Chao W, D'Amore PA. IGF2: epigenetic regulation and role in development and disease. Cytokine Growth Factor Rev. 2008;19(2):111-120.

41. Pollak M. Insulin and insulin-like growth factor signalling in neoplasia. Nat Rev Cancer. 2008; 8(12):915-928.

42. Fisher CE, Michael L, Barnett MW, Davies JA. Erk MAP kinase regulates branching morphogenesis in the developing mouse kidney. Development. 2001; 128(21):4329-4338.

43. Sparago A, Cerrato F, Vernucci M, Ferrero GB, Silengo MC, Riccio A. Microdeletions in the human H19 DMR result in loss of IGF2 imprinting and Beckwith-Wiedemann syndrome. Nat Genet. 2004; 36(9):958-960.

44. Prawitt D, et al. Microdeletion and IGF2 loss of imprinting in a cascade causing Beckwith-Wiedemann syndrome with Wilms' tumor. Nat Genet. 2005;37(8):785-786

45. Stark K, Vainio S, Vassileva G, McMahon AP. Epithelial transformation of metanephric mesenchyme in the developing kidney regulated by Wnt- 4 . Nature. 1994;372(6507):679-683.

46. Sim EU, et al. Wnt-4 regulation by the Wilms' tumour suppressor gene, WT1. Oncogene. 2002; 21(19):2948-2960.

47. Knudson AG Jr, Strong LC. Mutation and cancer: a model for Wilms' tumor of the kidney. J Natl Cancer Inst. 1972;48(2):313-324.

48. Lavik KJ, Schmittgen TD. Analysis of relative gene expression data using real-time quantitative PCR and the 2(-Delta Delta C(T)) Method. Methods. 2001; 25(4):402-408.

49. Hu Q, Lu JF, Luo R, Sen S, Maity S. Inhibition of $\mathrm{CBF} / \mathrm{NF}-\mathrm{Y}$ mediated transcription activation arrests cells at $\mathrm{G} 2 / \mathrm{M}$ phase and suppresses expression of genes activated at $\mathrm{G} 2 / \mathrm{M}$ phase of the cell cycle. Nucleic Acids Res. 2006;34(21):6272-6285.

50. Basson MA, et al. Sprouty1 is a critical regulator of GDNF/RET-mediated kidney induction. Dev Cell. 2005;8(2):229-239.

51 . Tibes $\mathrm{R}$, et al. Reverse phase protein array: validation of a novel proteomic technology and utility for analysis of primary leukemia specimens and hematopoietic stem cells. Mol Cancer Ther. 2006; 5(10):2512-2521.

52. Eisen MB, Spellman PT, Brown PO, Botstein D. Cluster analysis and display of genome-wide expression patterns. Proc Natl Acad Sci U S A. 1998; 95(25):14863-14868 